\title{
SÍNDROME DE DOWN: REVISIÓN SISTEMÁTICA SOBRE ESTUDIOS EFECTUADOS EN CHILE
}

\section{Down Syndrome: Systematic Review of Studies Carried out in Chile}

\author{
Marco Cossio-Bolaños \\ Universidad Católica del Maule, Talca. Chile \\ Rubén VIDAL-EsPINOZA \\ Universidad Católica del Maule, Talca. Chile \\ Universidad Católica Silva Henríquez, Santiago. Chile \\ José Sulla-Torres \\ Universidad Católica Santa María Arequipa, Arequipa. Perú \\ Camilo Urra-Albornoz \\ Universidad Santo Tomás, Talca. Chile \\ Catalina AcuñA \\ Universidad Católica del Maule, Talca. Chile \\ María DíAZ \\ Universidad Católica del Maule, Talca. Chile \\ Tamara GARRIDO \\ Universidad Católica del Maule, Talca. Chile \\ Daniela Herrera \\ Universidad Católica del Maule, Talca. Chile \\ Rossana Gómez-CAMPO \\ Universidad Católica del Maule, Talca. Chile \\ rossaunicamp@gmail.com
}

Recepción: 10 de junio de 2020

Aceptación definitiva: 16 de febrero de 2021 
SÍNDROME DE DOWN:

REVISIÓN SISTEMÁTICA SOBRE ESTUDIOS EFECTDUADOS EN CHILE

M. COSSIO, R. VIDAL, J. SUllA, C. URRA, C. ACUÑA, M. ' DÍAZ, T. GARRIDO, D. HERRERA Y R. GÓMEZ

Resumen: El objetivo del estudio fue identificar los tipos de estudios y las temáticas más investigadas en población con síndrome de Down en Chile y describir el tipo de muestra utilizada y el rango de edad durante el periodo de 2011 a 2020. Se efectuó un estudio documental (revisión sistemática). Se utilizó la base de datos Scopus para la búsqueda de información. Las palabras claves utilizadas fueron: Síndrome de Down y Chile. El periodo fue 2011 a 2020. La información se registró en una ficha de observación. Se identificaron 30 estudios. El 56,7 \% ( $n=17)$ son estudios descriptivos (transversales), el $10,0 \%(n=3)$ experimentales. Las temáticas de estudio en su mayoría abarcan temáticas sociales (8 estudios) y aspectos antropométricos (7 estudios), seguidas de variables fisiológicas, psicológicas, hematológicas, biomecánicas, pruebas prenatales y trastornos del sueño. El rango de edad de la muestra que utilizan los estudios va desde la etapa prenatal hasta 28 años y el tamaño de la muestra va desde uno hasta 1922 participantes. Los resultados del estudio sugieren la necesidad urgente de desarrollar estudios experimentales y longitudinales, la inclusión de adultos de edad media y avanzada, así como muestras en periodo prenatal, que permitan explorar temáticas relacionadas con variables fisiológicas, psicológicas, hematológicas, biomecánicas y pruebas de diagnóstico prenatal, puesto que son relevantes para la independencia y autonomía de las personas con síndrome de Down.

Palabras Clave: síndrome de Down; Chile; tipos de estudio.

Aвstract: The objective of the study was to identify the types of studies and the most investigated topics in the population with Down syndrome in Chile and to describe the type of sample used and the age range during the period from 2011 to 2020. A documentary study was carried out (systematic review). The Scopus database was used to search for information. The key words used were: Down Syndrome and Chile. The period was 2011 to 2020 . The information was recorded on an observation sheet. Thirty studies were identified. A total of $56,7 \%(n=17)$ were descriptive (cross-sectional) studies, $10,0 \%(n=3)$ were experimental studies. The study topics mostly cover social issues ( 8 studies) and anthropometric aspects ( 7 studies), followed by physiological, psychological, hematological, biomechanical, prenatal tests and sleep disorders. The age range of the sample used by the studies ranges from prenatal to 28 years and the sample size ranges from one to 1922 participants. The results of the study suggest the urgent need to develop experimental and longitudinal studies, the inclusion of middle-aged and older adults, as well as prenatal samples, to explore issues related to physiological, psychological, hematological, biomechanical variables and prenatal diagnostic tests, since they are relevant to the independence and autonomy of people with Down syndrome.

KEYwORDs: Down síndrome; Chile; study types.

\section{Introducción}

L sínDROME DE DOwn (SD) es la anomalía congénita no letal y de discapacidad
mental más frecuente en el ser humano, también conocido como trisomía del
par 21, que corresponde a una alteración genética producida por la presen- 
cia de un cromosoma extra en dicho par (Leonard, 2002; Eisermann, 2003; Parker, 2010; Dutta, 2012). Presenta un conjunto de características fisiológicas-anatómicas particulares como la hipotonía, hiperlaxitud articular y déficit sensorial que influyen en un retraso del desarrollo motor (Cabeza-Ruiz, 2016). Este síndrome requiere de prevención, diagnóstico, tratamiento oportuno e intervención en la salud en general.

$\mathrm{Su}$ incidencia a nivel internacional es 1/700 nacidos vivos. En Latinoamérica, la incidencia reportada de SD alcanza 1,88 casos por 1.000 nacidos vivos (Nazer y Cifuentes, 2011). En Chile se ha observado un cambio demográfico importante, caracterizado por un aumento de los nacimientos en mujeres de más de 40 años (Donoso, 2002), lo cual podría estar dado por la postergación de la maternidad y la ausencia de práctica del aborto eugenésico (Nazer, 2005) y un envejecimiento de las mujeres chilenas en edad fértil (Donoso y Carvajal, 2009).

Esto expone a un mayor riesgo de malformaciones congénitas y de hijos con síndrome de Down, llegando a una población de 25 niños con SD por cada 10.000 nacidos vivos, según los resultados del Estudio Colaborativo Latino Americano de Malformaciones Congénitas-ECLAMC1 (Nazer y Cifuentes, 2014).

Esta situación ubica a Chile como el país con mayor tasa de nacimiento de niños con esta condición en la región (Nazer y Cifuentes, 2011), a diferencia de países con alto diagnóstico prenatal y leyes de finalización del embarazo, donde la prevalencia ha disminuido en forma sostenida (Roizen y Patterson, 2003; Morris y Alberman, 2009; Cocchi et al., 2010).

El aumento de la población con SD en Chile es una orientación para los investigadores, por la importancia médica y social que esto conlleva, siendo una preocupación permanente de los diferentes programas de registro y monitoreo dedicados a estudiar este problema (Nazer y Cifuentes, 2011).

Esta situación genera un motivo para estar siempre alertas a cualquier cambio que se pueda producir en las prevalencias de niños, jóvenes y adultos con SD, por lo que es importante sistematizar los estudios primarios que se han efectuado en Chile, cuya información puede ayudar a profesionales e investigadores a identificar rangos de edades, temáticas de estudios y generar nuevas ideas de investigación para el beneficio de la población con SD en Chile.

En ese contexto, esta investigación documental prevé sistematizar las investigaciones que se han efectuado en niños, jóvenes y adultos con síndrome de Down y específicamente en la población chilena. Por lo que la información recabada podría ayudar a los investigadores e instituciones públicas y privadas a contextualizar, sondear y proyectar futuras investigaciones en temáticas y grupos etarios poco explorados.

Por lo tanto, el objetivo de esta investigación es identificar los tipos de estudios y las temáticas más investigadas en población con síndrome de Down en Chile, así como describir el tipo de muestra utilizada y el rango de edad durante el periodo de 2011 a 2020. 


\section{Metodología}

\subsection{Tipo de estudio}

Se efectuó una investigación documental (revisión sistemática) que permitió el estudio, la evaluación crítica y la síntesis de evidencias de investigaciones originales que se han desarrollado en Chile en niños y jóvenes con SD. Se adoptaron las recomendaciones propuestas en la guía Preferred Reporting Items for Systematic Reviews and Meta-Analyses (PRISMA), siguiendo los pasos: establecimiento del tema u objetivos de la revisión; establecimiento de los criterios de inclusión y exclusión de los artículos (selección de la muestra); categorización de los estudios; análisis de los resultados; discusión y presentación de los resultados (Mendes et al., 2008). El periodo de búsqueda de información se desarrolló entre enero a marzo del 2020.

\subsection{Tema y objetivos de la investigación}

Para poder describir los componentes relacionados con el problema identificado, se ha estructurado la siguiente pregunta de investigación: ¿Qué tipo de estudio, muestra y rango de edad incluidos en los estudios, y temáticas son consideradas en las investigaciones sobre el síndrome de Down en Chile? Como objetivos específicos esta revisión pretende caracterizar y analizar, de forma crítica y reflexiva, los tipos de estudio, la cantidad y rango de edad de la muestra y las temáticas estudiadas.

\subsection{Criterios de inclusión y exclusión}

Tras la lectura del texto completo, se seleccionaron los artículos según los criterios de inclusión: publicaciones a texto completo referidas a estudios primarios; publicaciones en inglés y español; estudios que fueron efectuados en el modelo humano. Se excluyeron los estudios que no cumplían los criterios anteriores, así como las revisiones bibliográficas, los comentarios, los resúmenes de conferencias y las disertaciones. Se añadirán, como criterios de exclusión, los estudios que incluyan como muestra a modelos animales.

\subsection{Estrategia de investigación}

Se efectuó la búsqueda de información en la base de datos Scopus, cuya temática está asociada al campo de las ciencias de la salud, educación, psicología y ciencias sociales. Fueron consideraron estudios publicados desde los años 2011-2020 en idioma inglés y español. Las palabras clave utilizadas fueron: Down's Syndrome (síndrome de Down) y Chile. 
(TITLE-ABS-KEY (“Down’s Syndrome”) AND (LIMIT-TO (AFFILCOUNTRY, “Chile”)) AND (LIMIT-TO (PUBYEAR, 2020) OR LIMIT-TO (PUBYEAR, 2019) OR LIMIT-TO (PUBYEAR, 2018) OR LIMIT-TO (PUBYEAR, 2017) OR LIMIT-TO (PUBYEAR, 2016) OR LIMIT-TO (PUBYEAR, 2015) OR LIMIT-TO (PUBYEAR, 2014) OR LIMIT-TO (PUBYEAR, 2013) OR LIMIT-TO (PUBYEAR, 2012) OR LIMIT-TO (PUBYEAR, 2011)).

\subsection{Procedimiento de selección del estudio}

El procedimiento de selección de estudios permite reducir los sesgos y los posibles errores humanos, asegurando que todos los artículos se seleccionan de la misma manera y garantizando la validez y veracidad de los resultados (Liberati et al., 2009). En la primera etapa se seleccionaron las publicaciones con título, resumen o cuerpo de texto relacionados con el tema del presente estudio y se eliminaron 13 publicaciones: 4 con un idioma diferente al definido en los criterios de inclusión, 6 por no ser estudios en Chile pero incluía un autor chileno y 3 por no permitir el acceso al texto completo. Después, se leyó cada publicación y se eliminaron 15 publicaciones según los criterios de elegibilidad de PRISMA descritos. De este análisis se seleccionaron 30 publicaciones. La Figura 1 muestra el diagrama de flujo que describe las fases del proceso de selección según el método PRISMA (Liberati et al., 2009).

Los principales motivos de la exclusión fueron no considerar el modelo humano en los estudios, no tener referencia de la edad de la muestra participante y ser una revisión de literatura. En el análisis de cada estudio fue recolectada información relativa al tipo de estudio utilizado, la cantidad de la muestra incluida, la edad de la muestra participante del estudio, así como el registro de la temática de estudio relacionada con el SD. La principal razón de exclusión fue el haber desarrollado el estudio en modelos animales.

Todo este procedimiento estuvo a cargo de dos investigadores del estudio, los que analizaron las 4 etapas por separado; finalmente, se llegó a un consenso para identificar los 30 estudios. 


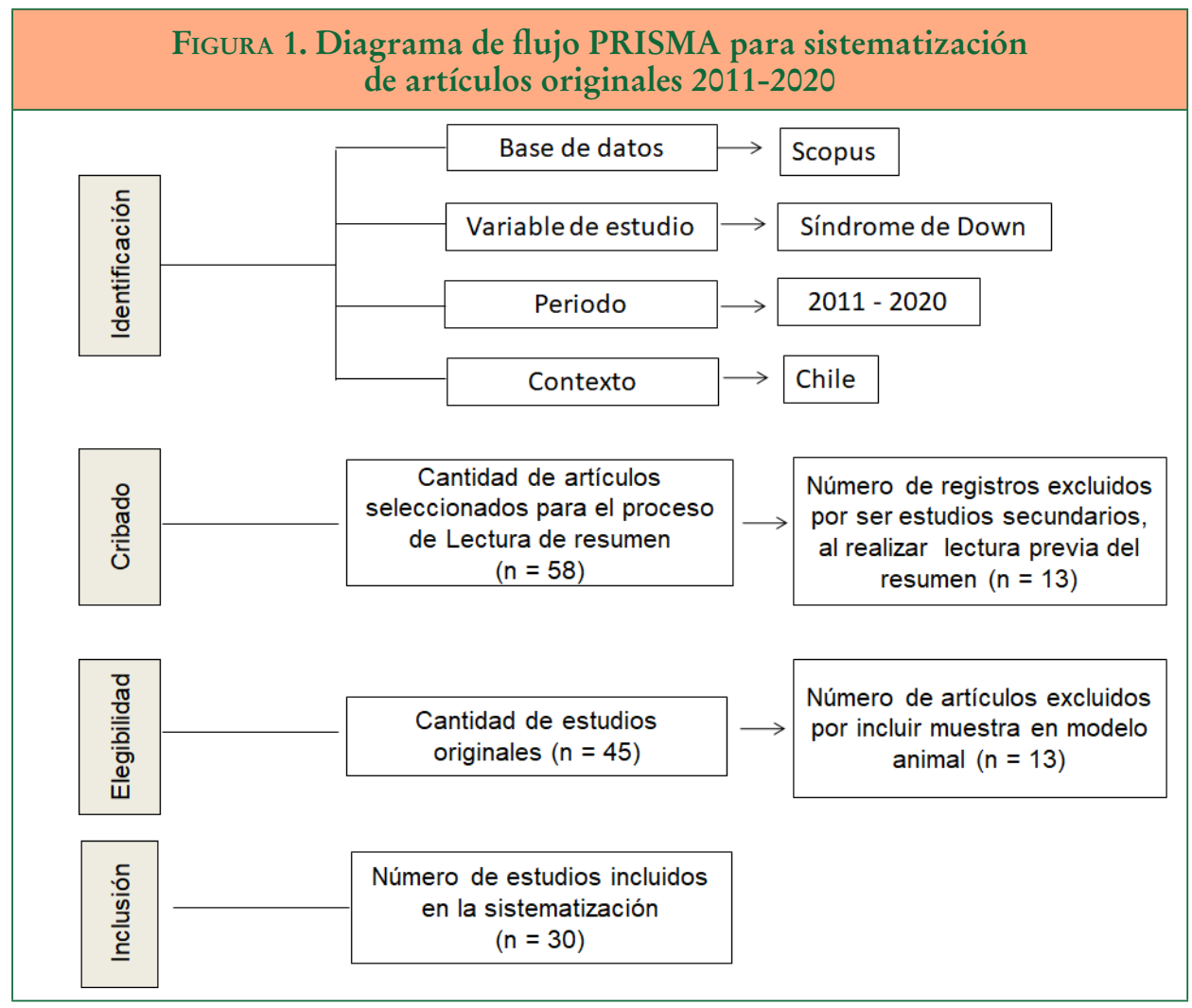

\subsection{Análisis de estudios}

El análisis de la síntesis de la revisión sistemática se basó en procedimientos cuantitativos y/o cualitativos. En el primer caso se organizaron los datos por medio de frecuencias, rango, porcentajes (\%) y en el segundo por medio de análisis de contenido de los indicadores considerados en la ficha de registro de información para analizar de forma interpretativa.

\section{Resultados}

En la Tabla 1 se muestran las características resumidas de los estudios sistematizados. Se han identificado un total de 30 estudios entre 2011 a 2020, de los cuales 14 son en inglés y 16 en español. Todos fueron digitados según el idioma, año y título de publicación. Fue durante el año 2018 donde se identificaron la mayor cantidad de estudios publicados $(\mathrm{n}=5)$. 


\begin{tabular}{|c|c|c|c|c|}
\hline \multicolumn{5}{|c|}{$\begin{array}{l}\text { TABLA 1. Investigaciones originales que se han efectuado en síndrome } \\
\text { de Down en Chile durante el periodo 2011- } 2020\end{array}$} \\
\hline N. ${ }^{\circ}$ & Autor & Años & Título & Idioma \\
\hline 1 & Sabat, C. et al. & 2020 & $\begin{array}{l}\text { Different abilities needed at home and school: The rela- } \\
\text { tion between executive function and adaptive behavior in } \\
\text { adolescents with Down syndrome }\end{array}$ & Inglés \\
\hline 2 & Gro, F. et al. & 2020 & $\begin{array}{l}\text { Hyperthyrotropinemia is common in preterm infants } \\
\text { who are born small for gestational age }\end{array}$ & Inglés \\
\hline 3 & $\begin{array}{l}\text { Guzmán, E. } \\
\text { et al. }\end{array}$ & 2019 & $\begin{array}{l}\text { Análisis de los sistemas sensoriales que contribuyen al } \\
\text { control postural en personas con síndrome de Down }\end{array}$ & Español \\
\hline 4 & Sabat, C. et al. & 2019 & $\begin{array}{l}\text { Adaptive Behavior and Intelligence in Adolescents With } \\
\text { Down Syndrome: An Exploratory Investigation }\end{array}$ & Inglés \\
\hline 5 & Linn, K. et al. & 2019 & $\begin{array}{l}\text { Development of communicative abilities in infants with } \\
\text { Down syndrome after systematized training in gestural } \\
\text { communication }\end{array}$ & Inglés \\
\hline 6 & Génova, L. et al. & 2018 & $\begin{array}{l}\text { Good health indicators in children with Down syndrome: } \\
\text { High frequency of exclusive breastfeeding at } 6 \text { months }\end{array}$ & Inglés \\
\hline 7 & Gómez, A. et al. & 2018 & $\begin{array}{l}\text { Efecto de una intervención basada en realidad virtual so- } \\
\text { bre las habilidades motrices básicas y control postural de } \\
\text { niños con Síndrome de Down }\end{array}$ & Español \\
\hline 8 & Gatica, P. et al. & 2018 & $\begin{array}{l}\text { Predictores de adiposidad corporal en jóvenes con síndro- } \\
\text { me de Down. }\end{array}$ & Español \\
\hline 9 & Arango, P. et al. & 2018 & $\begin{array}{l}\text { Developmental trajectories of children with Down syn- } \\
\text { drome by socio-economic status: the case of Latin Ame- } \\
\text { rica }\end{array}$ & Inglés \\
\hline 10 & Vega, V. et al. & 2018 & $\begin{array}{l}\text { Autodeterminación: Explorando las autopercepciones de } \\
\text { adultos con síndrome de Down chilenos }\end{array}$ & Español \\
\hline 11 & $\begin{array}{l}\text { Guzmán-Mu- } \\
\text { ñoz, E. et al. }\end{array}$ & 2017 & $\begin{array}{l}\text { Control postural en niños, adolescentes y adultos con sín- } \\
\text { drome de Down }\end{array}$ & Español \\
\hline 12 & $\begin{array}{l}\text { De la Piedra, M. } \\
\text { et al. }\end{array}$ & 2017 & $\begin{array}{l}\text { High frequency of dyslipidemia in children and adoles- } \\
\text { cents with Down Syndrome }\end{array}$ & Inglés \\
\hline 13 & Donoso, E. et al. & 2016 & $\begin{array}{l}\text { Aumento de la mortalidad infantil en niños con síndrome } \\
\text { de Down: Chile 1997-2013 }\end{array}$ & Español \\
\hline 14 & $\begin{array}{l}\text { Cárdenas, A. } \\
\text { et al. }\end{array}$ & 2016 & $\begin{array}{l}\text { Perfil de morbilidad en adolescentes chilenos con Síndro- } \\
\text { me de Down }\end{array}$ & Español \\
\hline 15 & $\begin{array}{l}\text { Brockmann, } \mathrm{P} \text {. } \\
\text { et al. }\end{array}$ & 2016 & $\begin{array}{l}\text { Sleep-disordered breathing in children with Down syn- } \\
\text { drome: Usefulness of home polysomnography }\end{array}$ & Inglés \\
\hline 16 & Paul, M. et al. & 2015 & $\begin{array}{l}\text { Perfil de morbilidad otorrinolarigológica en niños con } \\
\text { sindrome de Down }\end{array}$ & Español \\
\hline 17 & Venegas, E. et al. & 2015 & $\begin{array}{l}\text { Evaluación nutricional e indicadores de grasa visceral y } \\
\text { subcutánea en niños con Síndrome de Down }\end{array}$ & Español \\
\hline
\end{tabular}


SÍNDROME DE DOWN:

REVISIÓN SISTEMÁTICA SOBRE ESTUDIOS EFECTDUADOS EN CHILE

M. COSSIO, R. VIDAL, J. SULLA, C. URRA, C. ACUÑA, M. ${ }^{a}$ DÍAZ, T. GARRIDO, D. HERRERA Y R. GÓMEZ

\begin{tabular}{|c|c|c|c|c|}
\hline 18 & Jiménez, L. et al. & 2015 & $\begin{array}{l}\text { Malnutrición por exceso: alta frecuencia de sobrepeso y } \\
\text { obesidad en escolares chilenos con síndrome de Down }\end{array}$ & Español \\
\hline 19 & Lizama, M. et al. & 2015 & $\begin{array}{l}\text { Morbimortalidad hospitalaria en niños con Síndrome de } \\
\text { Down }\end{array}$ & Español \\
\hline 20 & Sirlopú, D. et al. & 2014 & $\begin{array}{l}\text { Actitudes implícitas y explícitas hacia personas con sín- } \\
\text { drome de Down: un estudio en colegios con y sin progra- } \\
\text { mas de integración en Chile }\end{array}$ & Español \\
\hline 21 & $\begin{array}{l}\text { Cresp-Barría, M. } \\
\text { et al. }\end{array}$ & 2014 & $\begin{array}{l}\text { Correlación de variables antropométricas como predictor } \\
\text { de salud, en una población de niños y adolescentes con } \\
\text { síndrome de Down de Temuco, Chile }\end{array}$ & Español \\
\hline 22 & Paul, M. A. et al. & 2013 & $\begin{array}{l}\text { ¿Cómo reciben los padres la noticia del diagnóstico de su } \\
\text { hijo con síndrome de Down? }\end{array}$ & Español \\
\hline 23 & Pérez, C. et al. & 2013 & Esotropia surgery in children with Down syndrome & Inglés \\
\hline 24 & $\begin{array}{l}\text { González, R. } \\
\text { et al. }\end{array}$ & 2013 & $\begin{array}{l}\text { Frontonasal Fold Thickness-to-Nasal Bone Length Ra- } \\
\text { tio as a Prenatal Sonographic Marker for Trisomy } 21 \text { in a } \\
\text { Low-Risk Population }\end{array}$ & Inglés \\
\hline 25 & Catalán, J. et al. & 2012 & $\begin{array}{l}\text { De Novo COL7A1 mutation in a patient with trisomy } \\
\text { 21: coexistence of dystrophic epidermolysis bullosa and } \\
\text { Down syndrome }\end{array}$ & Inglés \\
\hline 26 & Mosso, C. et al. & 2011 & $\begin{array}{l}\text { Evaluación de una intervención en actividad física en ni- } \\
\text { ños con síndrome de Down }\end{array}$ & Español \\
\hline 27 & Wong, A. et al. & 2011 & $\begin{array}{l}\text { Patient and provider attitudes toward screening for Down } \\
\text { syndrome in a Latin American country where abortion } \\
\text { is illegal }\end{array}$ & Inglés \\
\hline 28 & $\begin{array}{l}\text { Grinspon, R. } \\
\text { et al. }\end{array}$ & 2011 & $\begin{array}{l}\text { Early onset of primary hypogonadism revealed by serum } \\
\text { anti-Müllerian hormone determination during infancy } \\
\text { and childhood in trisomy } 2\end{array}$ & Inglés \\
\hline 29 & Nazer, J. et al. & 2011 & Estudio epidemiológico global del síndrome de Down & Español \\
\hline 30 & Ojeda, R. et al. & 2011 & $\begin{array}{l}\text { Correlación entre el Índice de Masa Corporal y Circunfe- } \\
\text { rencia de Cintura en una Muestra de Niños, Adolescentes } \\
\text { y Adultos con Discapacidad de Temuco - Chile }\end{array}$ & Inglés \\
\hline
\end{tabular}

En la Tabla 2 se observan los tipos de estudio y las temáticas publicadas en la base de datos Scopus. Se identificó que la mayoría de las investigaciones $(56,7 \%)$ fueron estudios descriptivos (trasversales) (17 estudios), seguidos de los estudios experimentales (3 estudios), que representan el 10,3\%. Las temáticas preferidas por los investigadores, en su mayoría, abarcan temáticas sociales (8 estudios, que representan el $26,7 \%$ ), aspectos antropométricos (6 estudios, representando el 20,0\%), seguidas de variables fisiológicas, psicológicas, hematológicas, biomecánicas, pruebas prenatales y trastornos del sueño. 
SÍNDROME DE DOWN:

REVISIÓN SISTEMÁTICA SOBRE ESTUDIOS EFECTDUADOS EN CHILE

M. COSSIO, R. VIDAL, J. SULlA, C. URRA, C. ACUÑA, M. ${ }^{a}$ DÍAZ, T. GARRIDO, D. HERRERA Y R. GÓMEZ

\begin{tabular}{|c|c|c|c|c|c|c|}
\hline \multicolumn{7}{|c|}{$\begin{array}{l}\text { TABLA 2. Tipos de estudios y temáticas desarrollados en Chile } \\
\text { en niños y jóvenes con síndrome de Down 2011-2020 }\end{array}$} \\
\hline \multirow{2}{*}{ Temáticas } & \multicolumn{6}{|c|}{ Tipos de estudio } \\
\hline & Experimental & Transversal & Longitudinal & Ex-Pos-Facto & Otros & Total \\
\hline Antropométricos & - & $4(13,3 \%)$ & - & $1(3,3 \%)$ & $1(3,3 \%)$ & $6(20,0 \%)$ \\
\hline Fisiológicos & $1(3,3 \%)$ & $2(6,7 \%)$ & - & $1(3,3 \%)$ & - & $4(13,3 \%)$ \\
\hline Hematológicos & - & $2(6,7 \%)$ & - & - & $1(3,3 \%)$ & $3(10,0 \%)$ \\
\hline Biomecánicos & - & $3(10,3 \%)$ & - & - & - & $3(10,0 \%)$ \\
\hline $\begin{array}{l}\text { Psicológicos (neu- } \\
\text { rocognitivos) }\end{array}$ & $1(3,3 \%)$ & $1(3,3 \%)$ & - & - & $2(6,7 \%)$ & $4(13,3 \%)$ \\
\hline Sociales & $1(3,3 \%)$ & $4(13,3 \%)$ & $1(3,3 \%)$ & $1(3,3 \%)$ & $1(3,3 \%)$ & $8(26,7 \%)$ \\
\hline T. sueño & - & $1(3,3 \%)$ & - & - & - & $1(3,3 \%)$ \\
\hline Pruebas prenatales & & & & & $1(3,3 \%)$ & $1(3,3 \%)$ \\
\hline Total & $3(10,0 \%)$ & $17(56,7 \%)$ & $1(3,3 \%)$ & $3(10,0 \%)$ & $6(20,0 \%)$ & $30(100 \%)$ \\
\hline
\end{tabular}

En relación con el rango de edad y el tamaño de la muestra, organizados por autores, se pueden observar en la Tabla 3. Se identificó que el rango de edad de la muestra que utilizan los estudios oscila en sujetos desde el nacimiento hasta los 28 años, además de una investigación que investigó en el periodo prenatal a mujeres gestantes que fueron sometidas a exámenes ecográficos. Con respecto al tamaño de la muestra, los estudios consideran entre uno hasta 1.922 participantes. Excepto un estudio que optó por efectuar una investigación con una base de datos de recién nacidos en hospitales chilenos extraída del Instituto de Estadística. 
SÍNDROME DE DOWN:

REVISIÓN SISTEMÁTICA SOBRE ESTUDIOS EFECTDUADOS EN CHILE

M. COSSIO, R. VIDAL, J. SULLA, C. URRA, C. ACUÑA, M. ${ }^{a}$ DÍAZ, T. GARRIDO, D. HERRERA Y R. GÓMEZ

\begin{tabular}{|c|c|c|c|}
\hline \multicolumn{4}{|c|}{$\begin{array}{l}\text { TABLA 3. Tamaño de la muestra y rango de edad utilizados en los estudios } \\
\text { efectuados en niños y jóvenes con SD en Chile 2011-2020 }\end{array}$} \\
\hline N. ${ }^{\circ}$ de publicación & Autor & $\mathrm{n}$ & Edad \\
\hline 1 & Sabat, C. et al. & 36 & 12 a 17 años \\
\hline 2 & Gro, F. et al. & 122 prematuros & neonatos \\
\hline 3 & Guzmán, E. et al. & 104 & 6 a 28 años \\
\hline 4 & Sabat, C. et al. & 53 & 12 a 17 años \\
\hline 5 & Linn, K. et al. & 24 & 18 a 22 meses \\
\hline 6 & Génova, L. et al. & 87 & 6 meses a 2 años \\
\hline 7 & Gómez, A. et al. & 16 & 3 a 10 años \\
\hline 8 & Gatica, P. et al. & 67 jóvenes & 10 y 25 años \\
\hline 9 & Arango, P. et al. & 105 niños & entre $15 \mathrm{y}$ meses \\
\hline 10 & Vega, V. et al. & 9 & 22 a 26 años \\
\hline 11 & Guzmán-Muñoz, E. et al. & 69 & 6 a 25 años \\
\hline 12 & De la Piedra, M. et al. & 218 & 2 a 18 años \\
\hline 13 & Donoso, E. et al. & Base de datos & 7 días a 11 meses \\
\hline 14 & Cárdenas, A. et al. & 67 pacientes & 10 a 20 años \\
\hline 15 & Brockmann, P. et al. & 44 & entre $0,1-10$ años \\
\hline 16 & Paul, M. et al. & 134 pacientes & 6 meses a 15 años \\
\hline 17 & Venegas, E. et al. & 40 & 3 a 13 años \\
\hline 18 & Jiménez, L. et al. & 81 & 6 a 8 años \\
\hline 19 & Lizama, M. et al. & 116 & Menores de 15 años \\
\hline 20 & Sirlopú, D. et al. & 80 & 11 a 15 años \\
\hline 21 & Cresp-Barría, M. et al. & 42 & 3 a 16 años \\
\hline 22 & Paul, M. A. et al. & 345 & No especifica \\
\hline 23 & Pérez, C. et al. & 44 & 8 meses a 17 años \\
\hline 24 & González, R. et al. & 1.922 & 16 y 32 semanas (prenatal) \\
\hline 25 & Catalán, J. et al. & 1 & 15 años \\
\hline 26 & Mosso, C. et al. & 18 & 5 a 9 años \\
\hline 27 & Wong, A. et al. & 107 & No especifica edad de gestantes \\
\hline 28 & Grinspon, R. et al. & 117 & 2 a 20 años \\
\hline 29 & Nazer, J. et al. & Indeterminado & Recién nacidos \\
\hline 30 & Ojeda, R. et al. & 123 hombres y 65 mujeres & 3 y 25 años \\
\hline
\end{tabular}

Ediciones Universidad de Salamanca / CC BY-NC-ND

Siglo Cero, vol. 52 (4), 2021, octubre-diciembre, pp. 155-172 
SÍNDROME DE DOWN:

REVISIÓN SISTEMÁTICA SOBRE ESTUDIOS EFECTDUADOS EN CHILE

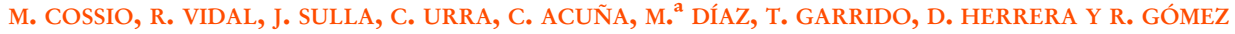

\section{Discusión}

Los resultados de la revisión sistemática han evidenciado que durante el periodo 2011 a 2020 las temáticas preferidas por parte de los investigadores fueron en su mayoría las relacionadas con aspectos sociales y antropométricos; además, los tipos de estudios que más destacan son los descriptivos transversales.

Estos hallazgos hacen reflexionar que las temáticas que implican variables fisiológicas, psicológicas, hematológicas, biomecánicas y pruebas prenatales aún son muy poco exploradas. Tal vez esta situación se deba a múltiples aspectos, entre los que se considera la falta de viabilidad en término de recursos financieros (Correa-García et al., 2012) que garanticen materializar los costos en el tiempo para desarrollar estudios en esas temáticas. Por ejemplo, el no contar con instalaciones y personal adecuadamente capacitado (Dubs de Moya, 2002) para el uso de equipos sofisticados, además de las limitaciones de tipo ético, que muchas veces conllevan la manipulación de variables y el incumplimiento de las directrices clínicas y de salud (Cummings et al., 2003), que posteriormente impiden la publicación de los resultados.

Asimismo, a pesar de ser solo un estudio identificado que aborda la temática específica relacionada con la aplicación de pruebas en periodo prenatal, resulta ser aún un tema poco explorado en Chile, sobre todo, si consideramos los hallazgos de un reciente estudio de revisión a nivel internacional que analizó la tendencia demográfica mundial del SD (Huete-García y Otaola-Barranquero, 2021), entre 1960 y 2019, donde muestra evidencia que existe una disminución de la incidencia de SD, que fue observada a través de la tasa de natalidad descendente, que se explica debido a que fueron aplicadas pruebas de detección prenatales realizadas por los diferentes sistemas de salud.

En cuanto a los tipos de investigación, se destaca que el mayor porcentaje de investigaciones en Chile en SD son las investigaciones descriptivas (transversales), probablemente esto se deba a que tienen como ventajas ser relativamente económicas y requieren poco tiempo para obtener resultados razonables a corto plazo (ÁlvarezHernández y Delgado-De la Mora, 2015). Estos estudios son muy útiles en la planeación y evaluación de intervenciones de la salud pública (Enarson et al., 2004; Hernández-Ávila et al., 2003; Levin, 2006). A su vez, enfrentan pocas dificultades éticas, dado que no existe una exposición deliberada por parte del investigador, en razón a que no se realiza un seguimiento de pacientes a lo largo del tiempo (Álvarez-Hernández y Delgado-De la Mora, 2015).

Asimismo, los estudios trasversales también presentan algunas limitaciones, que se explican debido a que la exposición y el resultado son examinados al mismo tiempo, existiendo una ambigüedad temporal en su relación, consecuentemente es difícil establecer inferencias causa-efecto (Aschengrau y Seage, 2003), por lo que, si se examina a la misma población en otro momento, los resultados obtenidos pueden ser diferentes, generando un "sesgo de memoria", especialmente si los datos cuestionados no son recordados con seguridad (Enarson et al., 2004; Hernández-Ávila et al., 2000; Mann, 2003; Levin, 2006). 
Por el contrario, los estudios experimentales implican un diseño de investigación que aporta la mejor evidencia para determinar si una intervención terapéutica, preventiva o educativa es eficaz o efectiva (Zurita-Cruz et al., 2018). Esto se puede conseguir cuando se cuenta con la subvención necesaria para el desarrollo de estudios, en razón al costo elevado que implica la propia rigidez de la intervención, sin dejar de lado las limitaciones de tipo ético y responsabilidad en la manipulación de variables.

Entre las principales desventajas de los estudios experimentales, se destaca que la población de estudio debe ser muy específica y definida (Manterola et al., 2019); además, en cuanto al control de las fuentes de invalidación interna, se destaca el riesgo de pérdida de muestra, debido a la adherencia de los participantes y la pérdida de interés y motivación durante la intervención.

Asimismo, en el caso de las investigaciones longitudinales, que incluye la existencia de medidas repetidas (más de dos) a lo largo de un seguimiento (Delgado Rodríguez y Llorca-Díaz, 2004), implican un mayor tiempo para su ejecución, teniendo que prestar mayor atención al control de calidad durante su ejecución, con el riesgo de producirse pérdida o abandono de muestra durante el seguimiento.

En suma, varios estudios trasversales, tras alcanzar sus resultados, sugieren la realización de investigaciones longitudinales y experimentales para confirmar sus resultados (Izquierdo-Gómez et al., 2017; Donoso y Vera, 2016; Gómez Álvarez et al., 2018). Pues las falencias y/o limitaciones encontradas pueden ser subsanadas en este tipo de investigaciones (longitudinales y experimentales). Esto podría ayudar a estudiar de una manera más amplia y concisa las temáticas, especialmente si se trata de verificar las relaciones de causa y efecto.

En cuanto a la muestra y el rango de edad que se han considerado en las investigaciones, podemos destacar que la mayor cantidad de estudios han incluido muestra desde el nacimiento, pasando por la infancia, adolescencia y juventud. Se identificaron estudios que incluyen como edad máxima los 28 años, además de un estudio que abarca el periodo prenatal. De hecho, al no haber estudios en Chile que abarquen edades superiores y solo uno en diagnóstico prenatal, se abren nuevas perspectivas para proyectar investigaciones en edades adultas en población con SD, así como en estudios en etapa prenatal que ayuden a conocer variables que podrían influir en la demografía del SD en Chile.

Cabe destacar que todas las etapas de la vida son relevantes para ser investigadas, sin embargo, en este estudio, la sistematización ha demostrado que se incluye en mayor porcentaje a menores de 28 años, pese a que, en los últimos años, las investigaciones a nivel mundial han demostrado que la población con síndrome de Down ha incrementado su promedio de vida (Saucedo-Rodríguez et al., 2017), estimándose por encima de los 60 años (Down España, 2011).

Estos cambios, tanto en la estructura como en el tamaño de la población de adulto mayor, generan mayores exigencias en su atención, especialmente hacia el desarrollo de estrategias que les permitan mejorar su calidad de vida; es así que las investigaciones realizadas hasta el momento son un punto de partida para estar preparados para enfrentar este nuevo desafío del incremento de la expectativa de vida. 
La evidencia mostrada en un estudio reciente (Huete-García y Otaola-Barranquero, 2021) sustenta la necesidad del registro específico de defectos congénitos, que se pueden conocer a través de la realización de pruebas prenatales, que ayudaría a reducir la población con $\mathrm{SD}$, fundamentándose en la asociación consistente que se encontró entre el diagnóstico prenatal y la tasa de natalidad. En ese sentido, aumentar las investigaciones en Chile, donde se registren pruebas prenatales, podría ser un camino para desarrollar la evaluación demográfica del SD en futuros estudios comparativos y de largo plazo.

En general, a pesar de que se han reflejado algunos estudios en población con SD durante el periodo 2011 a 2020, se destaca que estas investigaciones aún no son suficientes para atender la demanda del incremento de esta población en Chile, puesto que las temáticas más investigadas son desarrolladas en estudios transversales, centrados más en aspectos sociales y antropométricos.

En esencia, respecto a los resultados obtenidos en esta revisión, no fue posible encontrar similares estudios que permitan confrontar los hallazgos obtenidos con otros países y/o regiones, sin embargo, una revisión sistemática reciente que estudió la intervención temprana en SD (Robles-Bello y Sánchez-Teruel, 2019) resaltó que la mayoría de los estudios fueron realizados en América del Norte y en Europa en relación a otras regiones, además, las publicaciones son en idioma inglés y los diseños más utilizados fueron los estudios transversales. Estos hallazgos demuestran, de alguna forma, que Chile está en un proceso de crecimiento en investigación en poblaciones con SD, lo que puede servir de base para sensibilizar no solo al Gobierno, sino también a los investigadores.

En consecuencia, a través de los resultados obtenidos, es necesario desarrollar más investigaciones en Chile, especialmente, las que impliquen relaciones de causalidad. Por ejemplo, son escasos los estudios experimentales y longitudinales en relación a los diseños trasversales (17 estudios), por lo que estas evidencias deben ser elementos importantes para establecer prioridades entre los investigadores y para proponer objetivos y metas en la agenda del Gobierno e incorporar mecanismos que posibiliten el desarrollo de proyectos, investigaciones y políticas equitativas en todo el país.

Estudios futuros deben interesarse en desarrollar investigaciones que incluyan todas las fases etarias, con el intuito de generar estrategias que garanticen su atención y participación hacia los problemas relacionados con su discapacidad; a lo que se suma la necesidad de que el Gobierno también promueva la formulación y el desarrollo de proyectos de investigación en beneficio de la población con SD en las diferentes temáticas y edades poco exploradas. Todo esto, debido a que, en países como Chile, se presenta una gran necesidad de buscar conocimiento sobre el SD, pues, a pesar de ser un asunto estudiado por los científicos en algunas áreas, en la gran mayoría esos descubrimientos no llegan de forma correcta a aquellos profesionales que trabajan en el ambiente escolar y en las instituciones de salud.

De hecho, actualmente la nueva Ley de Inclusión en Chile (n. ${ }^{\circ}$ 20.845), promulgada en el 2015 (MINEDUC, 2015), está mucho más alineada con los principios de la educación inclusiva que sus leyes antecesoras, lo que permite aparentemente una educación inclusiva de calidad para todos los niños, jóvenes y adultos, respectivamente. 
SÍNDROME DE DOWN:

REVISIÓN SISTEMÁTICA SOBRE ESTUDIOS EFECTDUADOS EN CHILE

M. COSSIO, R. VIDAL, J. SULLA, C. URRA, C. ACUÑA, M. ${ }^{a}$ DÍAZ, T. GARRIDO, D. HERRERA Y R. GÓMEZ

Es necesario destacar algunas limitaciones del estudio, puesto que se utilizó una sola base de datos y fueron considerados estudios publicados únicamente en inglés y español, por lo que, al adicionar otras bases de datos e idiomas, es posible que se encuentren mayor número de estudios y otras temáticas no identificadas en este estudio. También, es necesario resaltar que es el primer estudio efectuado en Chile con estas características, lo que puede contribuir al planteamiento de nuevas líneas de investigación en poblaciones con SD.

\section{Conclusión}

Este estudio concluye que durante los años 2011 a 2020 en la base de datos Scopus se identificó un elevado número de investigaciones descriptivas (transversales) que consideran el estudio de población con SD en Chile. Las temáticas más investigadas están relacionadas con aspectos sociales y antropométricos enfocados sobre todo en población con menos de 28 años, así como en el periodo prenatal a través del estudio de mujeres gestantes entre 12 a 32 semanas. El tamaño de la muestra considerado en los estudios va desde uno hasta 1.922 participantes. Excepto un estudio que optó por efectuar una investigación con una base de datos extraída del Instituto de Estadística.

Estos resultados sugieren la necesidad urgente de desarrollar estudios experimentales y longitudinales, y la inclusión de adultos jóvenes y mayores en sus muestras, así como información sobre datos en etapa prenatal. Por otro lado, se deben explorar temáticas relacionadas con variables fisiológicas, hematológicas, biomecánicas, pruebas prenatales y psicológicas, puesto que podrían ayudar a conocer cómo otras variables y factores podrían impactar en la demografía del SD en Chile y son relevantes para la independencia y autonomía de las personas con síndrome de Down.

\section{Referencias bibliográficas}

Álvarez-Hernández, G. y Delgado-De la Mora, J. (2015). Diseño de estudios epidemiológicos. I. El estudio transversal: tomando una fotografía de la salud y la enfermedad. Boletín Clínico Hospital Infantil del Estado de Sonora, 32(1), 26-34.

Arango, P., Aparicio, A. y Tenorio, M. (2018). Developmental trajectories of children with Down syndrome by socio-economic status: the case of Latin America. Journal of Intellectual Disability Research, 62(9), 759-774.

Aschengrau, A. y Seage, Iit, G. (2003). Essentials of epidemiology in public health. Jones \&Bartlett Publishers.

Brockmann, P., Damiani, F., Núñez, F., Moya, A., Pincheira, E., Paul, M. y Lizama, M. (2016). Sleep-disordered breathing in children with Down syndrome: usefulness of home polysomnography. International Journal of Pediatric Otorbinolaryngology, 83, 47-50.

Cabeza-Ruiz, R., Castro-Lemus, N., Centeno-Prada, R. A. y Beas-Jiménez, J. D. (2016). Desplazamiento del centro de presiones en personas con síndrome de Down en bipedestación. Revista Andaluza de Medicina del Deporte, 9, 62-66. 
SÍNDROME DE DOWN:

REVISIÓN SISTEMÁTICA SOBRE ESTUDIOS EFECTDUADOS EN CHILE

M. COSSIO, R. VIDAL, J. SULLA, C. URRA, C. ACUÑA, M. ${ }^{a}$ DÍAZ, T. GARRIDO, D. HERRERA Y R. GÓMEZ

Cárdenas, A., María, P., Correa, C., Valderrama, S., Cerda, J. y Lizama, M. (2016). Perfil de morbilidad en adolescentes chilenos con síndrome de Down. Revista Médica de Chile, 144(8), 998-1005.

Catalán, J., Rodríguez, F., Yubero, M., Palisson, F., Gana, M., Kramer, S. y Repetto, G. (2012). De Novo Col7A1 mutation in a patient with trisomy 21: coexistence of dystrophic epidermolysis bullosa and Down syndrome. International Journal of Dermatology, $51,1078-1081$.

Cocchi, G., Gualdi, S. y Bower, C. (2010). International trends of Down syndrome 19932004 birth relation to maternal age and terminations of pregnancies. Birth Defects Research, $88,474-479$.

Correa-García, J. A., Arango-Serna, M. D. y Álvarez-Uribe, K. C. (2012). Metodología de valoración para proyectos de transferencia tecnológica universitaria. Caso aplicado - Universidad de Antioquia. Revista Facultad de Ciencias Económicas, 20(1), 91-106.

Cresp, M., CaAmaño Navarrete, F., Ojeda, R., Barría, C. y Jiménez, Á. (2014). Correlación de variables antropométricas como predictor de salud, en una población de niños y adolescentes con síndrome de Down de Temuco, Chile. Revista de la Facultad de Medicina, 62, 193-198.

Cummings, S. R., Browner, W. S. y Hulley, S. B. (2003). Elaborando a questão de pesquisa. En S. B. Hulley, S. R. Cummings, W. S. Browner, D. G. Grady y T. B. Newman, Delineando a pesquisa clínica: uma aordagem epidemiológica (pp. 35-41). 2. ${ }^{a}$ ed. Artmed.

Delgado-Rodríguez, M. y Llorca-Díaz, J. (2004). Estudios longitudinales: concepto y particularidades. Revista Española de Salud Pública, 78, 141-148.

De la Piedra, M. J., Alberti, G., Cerda, J., Cárdenas, A., Paul, M. A. y Lizama, M. (2017). Alta frecuencia de dislipidemias en niños y adolescentes con síndrome de Down. Revista Chilena de Pediatría, 88(5), 595-601.

Donoso, E., Becker, J. y Villarroel, L. (2002). Evolución de la natalidad y del riesgo reproductivo en mujeres de 40 o más años en la década de los 90. Revista Chilena de Obstetricia $y$ Ginecología, 67(2), 139-142.

Donoso, E., Carvajal, J. y Domínguez, M. A. (2009). Fecundity reduction and aging in fertile women population in the period 1990-2004 in Chile. Revista Médica de Chile, 137(6), 766-773.

Donoso, E. y Vera, C. (2016). Aumento de la mortalidad infantil en niños con síndrome de Down: Chile 1997-2013. Revista Médica de Chile, 144, 1432-1439.

Down España. (2011). Programa español de Salud para personas con Sindrome de Down. Down España 2011. Disponible en http://www.sindromedown.net/adjuntos/crublicaciones/90L_ downsalud.pdf

Dubs de Moya, R. (2002). El Proyecto Factible: una modalidad de investigación. Sapiens. Revista Universitaria de Investigación, 3(2), 1-18.

Dutta, U. R., Pidugu, V. K., Goud, V. y Dalal, A. B. (2012). Mosaic Downsyndrome with a marker: molecular cytogenetic characterization of the marker chromosome. Gene, 495, 199-204.

Eisermann, M. M., Delaraillère, A., Dellatolas, G., Tozzi, E., Nabbout, R., Dulac, O. y Chiron, C. (2003). Infantile spasms in Down syndrome-effects of delayed anticonvulsive treatment. Epilepsy Research, 55(1-2), 21-27.

Enarson, D. A., Kennedy, S. M. y Miller, D. L. (2004). Choosing a research study design and selecting a population to study. The International Journal of Tuberculosis and Long Disease, 8(9), 1151-1156. 
SÍNDROME DE DOWN:

REVISIÓN SISTEMÁTICA SOBRE ESTUDIOS EFECTDUADOS EN CHILE

M. COSSIO, R. VIDAL, J. SULLA, C. URRA, C. ACUÑA, M. ${ }^{a}$ DÍAZ, T. GARRIDO, D. HERRERA Y R. GÓMEZ

Gatica-Mandiola, P., Vidal-Espinoza, R., Gómez-Campos, R., Pacheco-Carrillo, J., Pino-Valenzuela, M. y Cossio-Bolaños, M. (2018). Predictores de adiposidad corporal en jóvenes con síndrome de Down. Revista Española de Nutrición Humana y Dietética, 24(4).

Génova, L., Cerda, J., Correa, C., Vergara, N. y Lizama, M. (2018). Buenos indicadores de salud en niños con síndrome de Down: alta frecuencia de lactancia materna exclusiva a los 6 meses. Revista Chilena de Pediatría, 89(1), 32-41.

Gómez Álvarez, N., Venegas Mortecinos, A., Zapata Rodríguez, V., López Fontanilla, M., Maudier Vásquez, M., Pavez-Adasme, G. y Hernández-Mosqueira, C. (2018). Efecto de una intervención basada en realidad virtual sobre las habilidades motrices básicas y control postural de niños con Síndrome de Down. Revista Chilena de Pediatría, 89(6), 747-752.

González, R., Aedo, S., Dezerega, V. y Sepúlveda, W. (2013). Frontonasal fold thicknessto-nasal bone length ratio as a prenatal sonographic marker for trisomy 21 in a low-risk population. Journal of Ultrasound in Medicine, 32(5), 795-800.

Grinspon, R., Bedecarrás, P., Ballerini, M., Íñiguez, G., Rocha, A., Resende, E., Brito, V., Milani, C., Gacitúa, V., Chiesa, A., Keselman, A., Gottlieb, S., Borges, M., Ropelato, M., Picard, J. Y., Codner, E. y Rey, R. (2011). Early onset of primary hypogonadism revealed by serum anti-Müllerian hormone determination during infancy and childhood in trisomy 21. International Journal of Andrology, 34, e487-98.

Grob, F., Gutiérrez, M., Leguizamón, L. y Fabres, J. (2020). Hyperthyrotropinemia is common in preterm infants who are born small for gestational age. Journal of Pediatric Endocrinology and Metabolism, 33(3), 375-382.

Guzmán-Muñoz, E., Méndez-Rebolledo, G., Villouta-Gutiérrez, O., Concha-Cisternas, Y. y Valdés-Badilla, P. (2019). Análisis de los sistemas sensoriales que contribuyen al control postural en personas con síndrome de Down. Neurología Argentina, 11(2), 75-80.

Hernández-Ávila, M., Garrido-Latorre, F. y López-Moreno, S. (2000). Diseño de estudios epidemiológicos. Salud Pública de México, 42(2), 144-154.

Huete-García, A. y Otaola-Barranquero, M. (2021). Demographic assessment of Down syndrome: a systematic review. International Journal of Environmental Research and Public Health, 18(1), 352.

Izquierdo-Gómez, R., Martínez-Gómez, D., Esteban-Cornejo, I., Hallal, P., GarcíaCervantes, L., Villagra, A. y Veiga, O. (2017). Changes in objectively measured physical activity in adolescents with Down syndrome: the UP\&Down longitudinal study: physical activity in Down syndrome. Journal of Intellectual Disability Research, 61(4).

Jiménez, L., Cerda, J., Alberti, G. y Lizama, M. (2015). Malnutrición por exceso: alta frecuencia de sobrepeso y obesidad en escolares chilenos con síndrome de Down. Revista Médica de Chile, 143, 451-458.

LEONARD, H. y Wen, X. (2002). The epidemiology of mental retardation: challenges and opportunities in the new millennium. Mental Retardation and Developmental Disabilities Research Reviews, 8(3), 117-134.

Levin, K. A. (2006). Study design Iiı: Cross-sectional studies. Evidence-Based Dentistry, 7, 24-25.

Liberati, A., Altman, D. G., Tetzlaff, J., Mulrow, C., Gøtzsche, P. C., Ioannidis, J. P., Clarke, M., Devereaux, P. J., Kleijnen, J. y Moher, D. (2009). The Prisma statement for reporting systematic reviews and meta-analyses of studies that evaluate health care interventions: explanation and elaboration. PLoS Medicine, 6(7), e1000100. 
SÍNDROME DE DOWN:

REVISIÓN SISTEMÁTICA SOBRE ESTUDIOS EFECTDUADOS EN CHILE

M. COSSIO, R. VIDAL, J. SULLA, C. URRA, C. ACUÑA, M. ${ }^{a}$ DÍAZ, T. GARRIDO, D. HERRERA Y R. GÓMEZ

Linn, K., Sevilla, F., Cifuentes, V., Eugenin, M. I., Río, B., Cerda, J. y Lizama, M. (2019). Desarrollo de habilidades comunicativas en lactantes con síndrome de Down posterior a capacitaciones sistematizadas en comunicación gestual. Revista Chilena de Pediatría, 90(2), 175-185.

Lizama, M., Cerda, J., Monge, M., Carrillo-Mayanquer, I., Clavería-Rodríguez, C. y Castillo-Moya, A. (2016). Morbimortalidad hospitalaria en niños con síndrome de Down. Revista Chilena de Pediatría, 87(2), 102-109.

Lizama, M., Retamales, N. y Mellado, C. (2013). Recomendaciones de cuidados en salud de personas con síndrome de Down: 0 a 18 años. Revista Médica de Chile, 141(1), 80-89.

Mann, C. J. (2003). Observational research methods. Research design II: cohort, cross sectional and case-control studies. Emergency Medicine Journal, 20, 54-60.

Manterola, C., Quiroz, G., Salazar, P. y García, N. (2019). Metodología de los tipos y diseños de estudio más frecuentemente utilizados en investigación clínica. Revista Médica Clínica Las Condes, 30(1), 36-49.

Mendes, K., Silveira, R. y Galvão, C. (2008). Revisão integrativa: método de pesquisa para a incorporação de evidências na Saúde e na Enfermagem. Texto and Contexto - Enfermagem, $17,758-764$.

Ministerio De Educación [Mineduc]. (2015). Ley Núm. 20.845 de Inclusión Escolar que regula la admisión de los y las estudiantes, elimina el financiamiento compartido y prohíbe el lucro en establecimientos educacionales que reciben aportes del Estado. Promulgación: 29-mayo-2015. Santiago, Chile, Mineduc. Disponible en: http://bcn.cl/1uv1u

Morris, J. K. y Alberman, E. (2009). Trends in Down's syndrome live births and antenatal diagnoses in England and Wales from 1989 to 2008: analysis of data from the National Down Syndrome Cytogenetic Register. BMJ, 339, b3794.

Mosso, C., Santander, P., Pettinelli, P., Valdez, M., Celis, M., Espejo, F., Navarro, L. y Sepúlveda, F. (2011). Evaluación de una intervención en actividad física en niños con síndrome de Down. Revista Chilena de Pediatría, 82(4), 311-318.

Nazer, H. J., Aguila, R. A. y Cifuentes, O. L. (2006). Increasing rates of Down syndrome among newborns in Chile from 1972 to 2005. Revista Médica de Chile, 134(12), 1549-1557.

NAzer, J. y Cifuentes, L. (2011). Estudio epidemiológico global del Síndrome de Down. Revista Chilena de Pediatría, 2(82), 105-112.

Nazer, J. y Cifuentes, L. (2014). Prevalencia al nacimiento de malformaciones congénitas en las maternidades chilenas participantes en el ECLAMC en el período 2001-2010. Revista Médica de Chile, 140(9), 1150-1156.

Ojeda Nahuelcura, R. y Cresp Barría, M. (2011). Correlación entre Índice de Masa Corporal y Circunferencia de Cintura en una muestra de niños, adolescentes y adultos con discapacidad de Temuco, Chile. International Journal of Morphology, 29(4), 1326-1330.

Parker, S. E., Mai, C. T., Canfield, M. A., Rickard, R., Wang, Y., Meyer, R. E., Anderson, P., Mason, C. A., Collins, J. S., Kirby, R. S., Correa, A. y National Birth Defects Prevention Network. (2010). Updated national birth prevalence estimates for selected birth defects in the United States, 2004-2006. Birth Defects Research. Part A, Clinical and Molecular Teratology, 88(12), 1008-1016.

Paul, M., Bravo, A., Beltrán, C., Cerda, J., Angulo, D. y Lizama, M. (2015). Perfil de morbilidad otorrinolaringológica en niños con síndrome de Down. Revista Chilena de Pediatría, 86(5), 318-324.

Paul, M., Cerda, J., Correa, C. y Lizama, M. (2013). ¿Cómo reciben los padres la noticia del diagnóstico de su hijo con síndrome de Down? Revista Médica de Chile, 141(7), 879-886. 
Pérez, C., Zuazo, F., Zanolli, M., Guerra, J., Acuña, O. e Iturriaga, H. (2013). Esotropia surgery in children with Down syndrome. Journal of American Association for Pediatric Ophthalmology and Strabismus \{JAAPOS\}, 17(5), 477- 479.

Robles-Bello, M. A. y SÁnchez-Teruel, D. (2019). Intervención temprana en síndrome de Down: una revisión sistemática. Revista Iberoamericana de Psicología, 12(3), 55-70.

Roizen, N. J. y Patterson, D. (2003). Down's syndrome. Lancet, 361(9365), 1281-1289.

Sabat, C., Arango, P., Tassé, M. y Tenorio, M. (2020). Different abilities needed at home and school: the relation between executive function and adaptive behaviour in adolescents with Down syndrome. Scientific Reports, 10(1). https://doi:10.101038/s41598-020-58409-5

Sabat, C., Tassé, M. y Tenorio, M. (2019). Adaptive behavior and intelligence in adolescents with Down syndrome: an exploratory investigation. Intellectual and Developmental $\mathrm{Di}$ sabilities, 57(2), 79-94.

Saucedo-Rodríguez, J., Cruz-Ortiz, M., Pérez-Rodríguez, M. y Vega-Cordova, V. (2017). Envejecimiento de las personas con síndrome de Down: un nuevo reto para la salud. Index de Enfermería, 26(3), 166-169.

Sirlopú, D., González, R., Bohner, G., Siebler, F., Millar, D., Ordóñez, G., Torres, D. y De Tezanos-Pinto, P. (2012). Actitudes implícitas y explícitas hacia personas con síndrome de Down: un estudio en colegios con y sin programas de integración de Chile. Revista de Psicología Social, 27, 199-210.

Vega, V., Álvarez, I. y Jenaro Río, C. (2018). Autodeterminación explorando las autopercepciones de adultos con síndrome de Down chilenos. Siglo Cero, 49(2), 89-104.

Venegas, E., Ortiz, T., Grandfeltd, G., Zapata, D., Fuenzalida, P. y Mosso, C. (2015). Evaluación nutricional e indicadores de grasa visceral y subcutánea en niños con síndrome de Down. Revista Médica Internacional sobre el Síndrome de Down, 19(2), 21-27.

Wong, A., Kuppermann, M., Creasman, J., Sepúlveda, W. y Vargas, J. (2011). Patient and provider attitudes toward screening for Down syndrome in a Latin American country where abortion is illegal. International Journal of Gynecology and Obstetrics, 115, 235-239.

Zurita-Cruz, J., Márquez-González, H., Miranda-Novales, M. y Villasís-Keever, M. (2018). Estudios experimentales: diseños de investigación para la evaluación de intervenciones en la clínica. Revista Alergia México, 65, 178. 